\section{Sex-influenced autosomal dominant optic atrophy is caused by mutations of IVS9 $+2 A>G$ in the OPA1 gene}

\section{To the Editor:}

Autosomal dominant optic atrophy (DOA) is one of the most common genetic causes of vision loss. Clinically, it is characterized by progressive vision loss, color vision anomalies, and temporal pallor of the optic disc. Two loci have been previously mapped to be associated with DOA. OPA1 is located on chromosome 3q28. Another locus for DOA was mapped to chromosome 18. However, this gene is not yet identified.

We previously reported two large families, Family A and Family B with DOA. ${ }^{1}$ In Family A, 17 individuals have diminished vision acuity ranging from $20 / 25$ to $20 / 800$. Vision loss among affected males was much more severe than in females, suggesting sex-influenced phenotypes. Genotyping with microsatellite markers flanking OPA1 result in a maximum LOD score of 4.68 at $=0.0$. However, the mutations were not found in OPA1 by single-strand conformation polymorphism. Figure 1.

Here, we performed direct sequencing of OPA1 in Family A. We identified a mutation IVS9 $+2 \mathrm{~A}>\mathrm{G}$. This mutation was previously reported in one sporadic case. The mutation causes in-frame skipping of exon 9 and loss of 20 amino acids, ${ }^{2}$ which is part of the GTPS domain of OPA1.

IVS9 $+2 \mathrm{~A}>\mathrm{G}$ mutation can cause sex-influenced optic atrophy. Future studies will expand our understanding of phenotype-genotype correlations and sex-influenced phenotype observed.

\author{
Taosheng Huang, MD, PhD \\ Jong Dae Whang, MD, PhD \\ Department of Pediatrics \\ University of California \\ Irvine, California \\ Virginia Kimonis, MD \\ Harvard Medical School, \\ Boston, Massachusetts
}

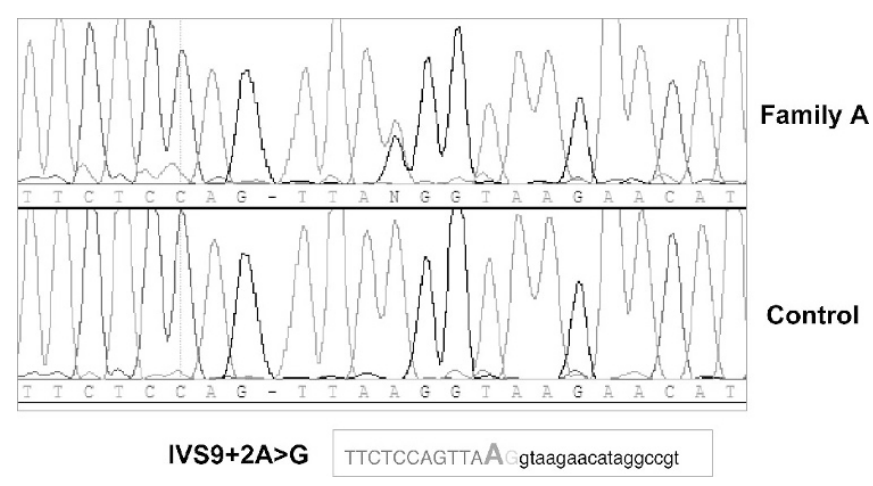

Fig. 1 Mutation detected in Family A. A to G mutation at nucleotide 983, which is also the exon 9 splice donor site (IVS9 $+2 \mathrm{~A}>\mathrm{G}$ ). Exon sequences are in upper case; intron sequences are in lower case.

\section{ACKNOWLEDGMENTS}

We thank the patients and families for participating in our study and the physicians for referring the patients to us. This research is support by the General Clinical Research Center at University of California, Irvine.

\section{References}

1. Chen AS, Kovach MJ, Herman K, Avakian A et al. Clinical heterogeneity in autosomal dominant optic atrophy in two 3q28-qter linked central Illinois families. Genet Med 2000;2(5):283-289.

2. Baris O, Delettre C, Amati-Bonneau P, Surget MO et al. Fourteen novel OPAl mutations in autosomal dominant optic atrophy including two de novo mutations in sporadic optic atrophy. Hum Mutat 2003;21(6):656. 